\title{
Modeling and characterization of three kinds of MEMS resonators fabricated with a thick polysilicon technology
}

\author{
D. Paci · M. Mastrangeli • A. Nannini · F. Pieri
}

Received: 30 June 2005 / Revised: 30 November 2005 / Accepted: 22 December 2005

(C) Springer Science + Business Media, LLC 2006

\begin{abstract}
Three different kinds of two-port flexural resonators, with both clamped and free ends, and with nominal resonance frequencies between $5 \mathrm{MHz}$ and $50 \mathrm{MHz}$, were designed and fabricated. Among them, a novel free-free third-mode resonator, as well as a tunable free-free resonator, designed to maintain a high quality factor despite its tunability, are presented. Because of reduced energy loss in the clamps, higher quality factors are expected from free-free devices.

To estimate the resonators performance, the effect of temperature and axial stresses on the resonators is investigated: for the clamped-clamped resonator, a theoretical model is also presented. FEM simulations are performed for the three geometries and the results are discussed.
\end{abstract}

Keywords MEMS resonators - FEM simulations · Temperature stability $\cdot$ Residual stress $\cdot$ Tunable MEMS resonators.

\section{Introduction}

MEMS resonators have been proposed as fundamental components for RF filters, mixers and oscillators [1]. The use of such devices as frequency references for RF communication systems is very attractive. Their very high quality factor $[2,3]$ implies a very low phase noise for oscillators including a MEMS resonator as the frequency selective element,

D. Paci $(\bowtie)$

IEIIT-Sezione di Pisa, CNR, Via Caruso,

56122 Pisa, Italy

e-mail: dario.paci@iet.unipi.it

D. Paci · M. Mastrangeli · A. Nannini · F. Pieri

Dipartimento di Ingegneria dell'Informazione, Università di Pisa,

Via Caruso, 56122 Pisa, Italy which, in turns, implies a very high short term stability [2]. To evaluate their overall performance as frequency references, many studies about long term stability of the resonance frequency [4] and its dependence on the temperature [5, 6] have been carried out. Also, their sensitivity to fluctuations of the fabrication process parameters is of great importance in the same respect. Specifically, the residual stress acting on the beam, which mainly depends on the deposition conditions of the material which constitutes the resonator, is expected to influence the resonance frequency of nominally identical resonators, either from different chips or from the same chip.

In this paper three different types of two-port flexural resonators with lateral driving and sensing are considered: a classic clamped-clamped beam, resonating on its first resonance mode [7] (type CC1), a free-free beam resonating on the first mode with matched flexural support beams [6] (type FF1), and a free-free beam resonating on the third mode with matched flexural support beams (type FF3). This last device is similar to the one presented in [8], but in that case the matched supports were torsional rods.

In the following, FEM simulations performed with FEMLAB ${ }^{\circledR}[9]$ on the three geometries are presented, with emphasis on the aforementioned effects (temperature and residual stress) on the resonance frequencies. A theoretical analysis of the expected behavior of the $\mathrm{CC} 1$ resonator is presented. Closed form analytical expression for the frequency shift is derived and compared with the simulation results. Basic operation of the fabricated devices is validated with measurements of the frequency response of FF1 and FF3 devices.

\section{Design and fabrication}

The resonators were fabricated by using the THELMA MEMS technology, developed at STMicroelectronics. 
THELMA is a surface micromachining process developed to fabricate capacitive inertial sensors. In the process flow a first polysilicon thin layer, used for electrical connectivity, is followed by a silicon dioxide sacrificial layer, and by a second, thick, epitaxially grown polysilicon layer, which constitutes the structure of the presented resonators and of their driving electrodes. Selective etching of the silicon dioxide releases the thick polysilicon structures, which become free to move under electrostatic forces. The high epitaxial polysilicon thickness implies a large capacitance between electrodes, and thus a large electromechanical coupling [10]. The resonating element is a flexural beam, which is electrostatically coupled to two symmetrical electrodes, one used to drive the beam into resonance, the other to collect the output current signal. SEM pictures of CC1, FF1, and FF3 resonators are presented in Figs. 1-3, respectively. The CC1 resonator is obtained simply by clamping the ends of the beam to the substrate. The free-free condition, instead, is obtained [6] by suspending the resonating beam by two doubly clamped support beams, joined to the main beam in two points which correspond to the nodes of the free-free mode which has to be excited. The length of the support beams of both FF1 and FF3 devices is designed so that they resonate on their second clamped-clamped mode at the same frequency of the main beam. As a consequence, at least ideally, they do not exert any bending moment on the main beam, so that the main beam is minimally affected by supports and anchors; basically, the supports are used to decouple the main beam from the anchors. This design choice should reduce the so called "anchor losses", which are generally considered as limiting mechanism for the quality factor of RF MEMS

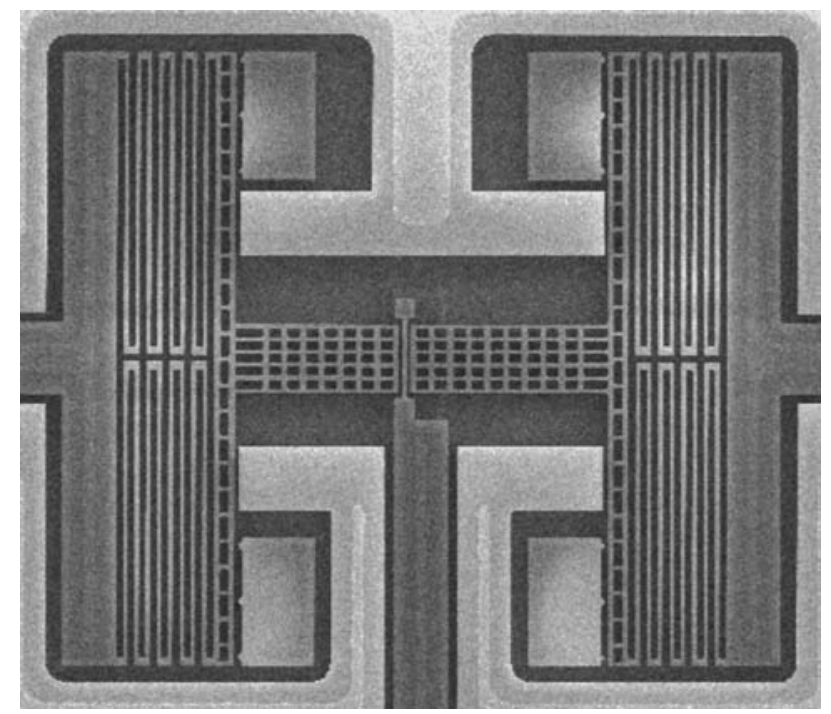

Fig. 1 Clamped-clamped resonator on the first resonance mode (type $\mathrm{CC} 1$ ). The nominal resonance frequency is $10 \mathrm{MHz}$. The large structures on the right and the left are the electrostatic micro-motors used to reduce the electrode/resonator gap

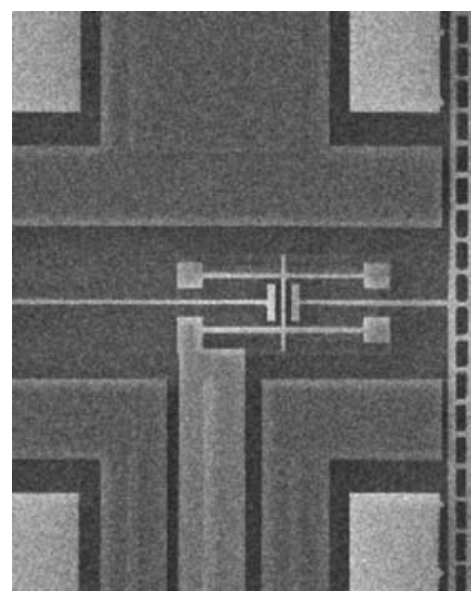

Fig. 2 Free-free resonator on the first resonance mode (type FF1). The nominal resonance frequency is $10 \mathrm{MHz}$

Fig. 3 Free-free resonator on the third resonance mode (type FF3). The nominal resonance frequency is $10 \mathrm{MHz}$
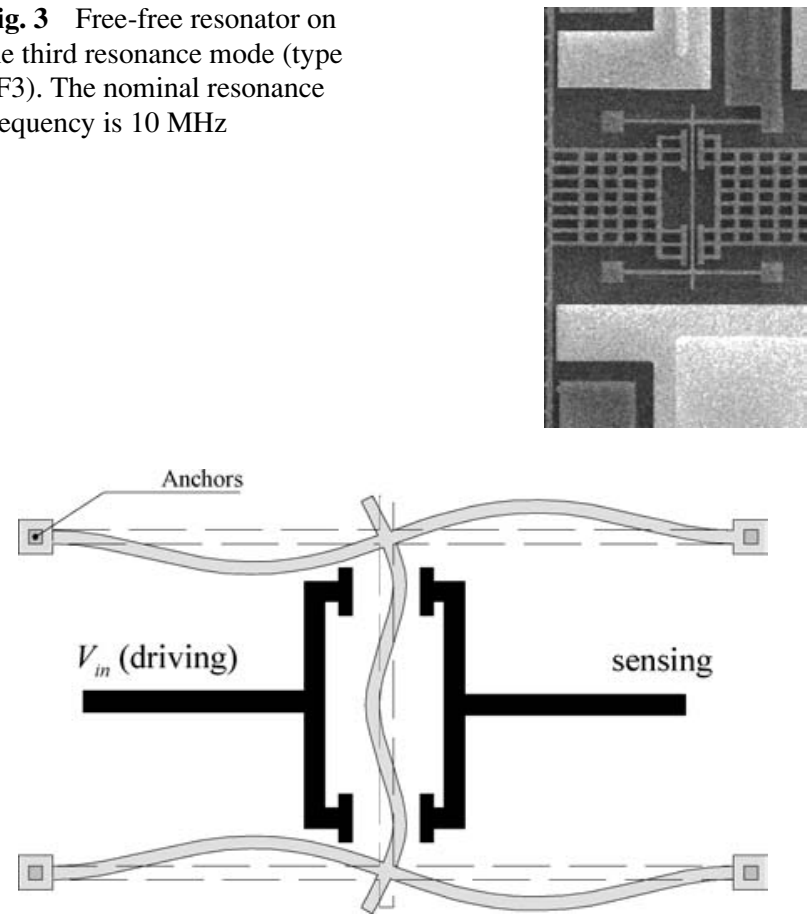

Fig. 4 Schematic structure for a free-free third mode (FF3) resonator, with flexural supports

resonators in vacuum [3]. The electrodes were shaped to obtain a high electro-mechanical coupling for the desired (1st or 3rd) mode-shape, i.e. they were placed facing the main beam where the amplitude of the deflected shape is expected to be larger. For the same reason, a low coupling with the other (parasitic) modes is also expected. The geometry of a FF3 resonator is shown in Fig. 4, where the electrodes and the deflected shape are also represented.

For higher order modes resonators, as the FF3 type, improved performances are expected. Specifically, we note that a FF3 resonator main beam is longer than the one of a FF1 resonator at the same resonance frequency, and the 
consequent larger electrode area means a larger electromechanical coupling between the electrode and the main resonator beam. As a consequence, the actuation and sensing mechanisms are more efficient, and this leads to reduced insertion losses, (if the same qualify factor is supposed for the two types). A rigorous analysis of this effect, performed by using the theoretical approach developed in [11], confirms the above qualitative explanation.

To maximize the electromechanical coupling between the electrodes and the resonator, the gap between them must be kept as small as possible [11]. To overcome the resolution limit of the process, the electrodes were mounted on electrostatic micro-motors to drive them closer to the beam during operation [7].

The resonance frequency of the fabricated devices can be tuned by changing the bias voltage $V_{\text {pol }}[6,11]$, but for the free-free resonators the tuning produces a decrease of the quality factor $Q$ [6]. A possible reason of this behavior can be the mismatch in the resonance frequencies of the main beam and the supports. In fact, the bias voltage is commonly applied only between the driving/sensing electrodes and main beam, so that the main beam is affected by a change in its equivalent stiffness due to the electromechanical coupling [11], while the supports are not; in this case the assumption of no moment exerted by the supports on the main beam at resonance is not valid anymore. A possible solution is given by the device schematically represented in Fig. 5: the same frequency change is forced both on the beam and on the supports, by placing a couple of electrodes in front of the supports and by biasing them with a proper DC voltage $V_{\text {tune }}-V_{\text {pol }}$ with respect to them.

\section{Temperature and axial stress effect on clamped-clamped resonators}

The analytical derivation of the resonance frequencies for axially loaded clamped-clamped beams has been already

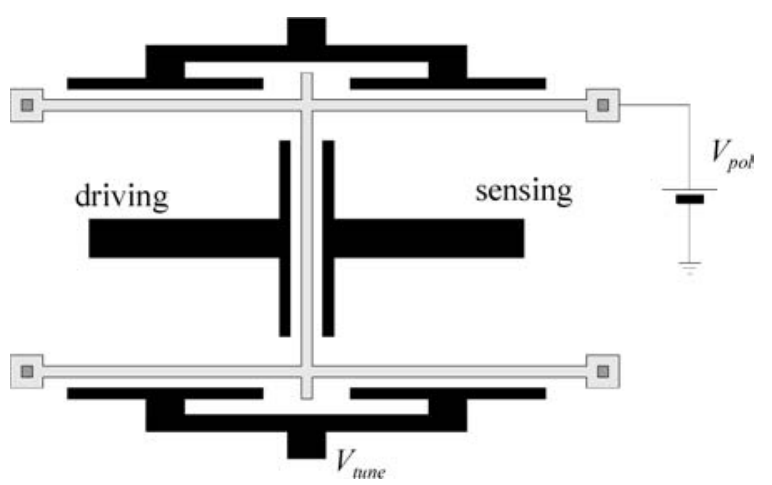

Fig. 5 Schematic structure for a free-free resonator on the first resonance mode, with two lateral electrodes to stabilize the quality factor with respect to the DC bias voltage carried out in [12]. Our approach is slightly different and leads to a closed form for the resonance frequencies. The effect of temperature and/or residual stress on $\mathrm{CC}$ resonators can be modeled by supposing a uniform stress parallel to the beam axis, and by including its effect on the equation of motion of the beam. The total compressive axial stress $\sigma_{e q}$ can be caused by the fabrication residual stress $\sigma_{R}$, by the temperature difference between the beam and the substrate, and by the difference between their respective thermal expansion coefficients. Consequently its expression can be written as:

$\sigma_{e q}=\sigma_{R}+E\left[\alpha_{m} \Delta T+\left(T_{m}-T_{\text {ref }}\right) \Delta \alpha\right]$

where $\alpha_{m}$ is the average value of the thermal expansion coefficients and $\Delta \alpha$ their difference; $T_{m}$ is the mean temperature of the resonator and the substrate, $\Delta T$ their difference and $T_{\text {ref }}$ a reference temperature; $E$ is the Young's modulus of the material.

To obtain the resonance frequencies of a $\mathrm{CC}$ beam, the Euler-Bernoulli equation for a beam with rectangular section under axial load has to be solved. The resonance frequencies of the structure are given by:

$f_{n}=\frac{1}{2 \pi}\left(\frac{\lambda_{n}}{L}\right)^{2} \sqrt{\frac{E}{12 \rho}} W$

where $\rho$ is the volumetric mass density of the resonator, while $W$ and $L$ are the width and the length of the resonator, respectively; $\lambda_{n}$ is the $n$th eigenvalue of the eigenvalue equation [12]:

$1-\cos \left(\lambda_{1 n}\right) \cosh \left(\lambda_{2 n}\right)=\frac{\lambda_{1 n}^{2}-\lambda_{2 n}^{2}}{2 \lambda_{1 n} \lambda_{2 n}} \sin \left(\lambda_{1 n}\right) \sinh \left(\lambda_{2 n}\right)$

with:

$\lambda_{1 n}=\sqrt{\frac{\sqrt{4 \lambda_{n}^{4}+\left(\frac{12 \sigma_{e q} L^{2}}{E W^{2}}\right)^{2}}+\frac{12 \sigma_{e q} L^{2}}{E W^{2}}}{2}}$

and:

$\lambda_{2 n}=\sqrt{\frac{\sqrt{4 \lambda_{n}^{4}+\left(\frac{12 \sigma_{e q} L^{2}}{E W^{2}}\right)^{2}}-\frac{12 \sigma_{e q} L^{2}}{E W^{2}}}{2}}$

If the axial load is small enough, it is possible to obtain, after straightforward algebraic manipulation, an expression for the $n$th resonance frequency $f_{n}$ :

$f_{n}=f_{n}^{0}\left[1-\frac{3}{\pi W f_{n}^{0}} \frac{G\left(\lambda_{n}^{0}\right)}{\sqrt{12 E \rho}} \sigma_{e q}\right]$ 
where $f_{n}^{0}$ is the $n$th resonance frequency of the unstressed structure, $\lambda_{n}^{0}$ the eigenvalue of the unstressed beam, and $G\left(\lambda_{n}^{0}\right)$ is a function only of the eigenvalue $\lambda_{n}^{0}$ and of the mode-shape of the unstressed structure:

$$
\begin{aligned}
& G\left(\lambda_{n}^{0}\right) \\
& =\frac{\lambda_{n}^{0}\left(\cosh \lambda_{n}^{0} \sin \lambda_{n}^{0}+\cos \lambda_{n}^{0} \sinh \lambda_{n}^{0}\right)-2 \sinh \lambda_{n}^{0} \sin \lambda_{n}^{0}}{\lambda_{n}^{0}\left(\cosh \lambda_{n}^{0} \sin \lambda_{n}^{0}-\cos \lambda_{n}^{0} \sinh \lambda_{n}^{0}\right)}
\end{aligned}
$$

$G\left(\lambda_{n}^{0}\right)$ increases with $n$ and it is nearly 1 for $n>5$. For comparison, $\gamma_{n}$ of ref. [12] equals our $-12 G\left(\lambda_{n}^{0}\right) /\left(\lambda_{n}^{0}\right)^{2}$.

The stability of $f_{n}$ descends at once from (4). For example, its value with respect to the temperature difference $\Delta T$ between resonator and substrate is:

$S=\frac{1}{f_{n}^{0}} \frac{\partial f_{n}}{\partial \Delta T}=-\frac{3 \alpha_{m}}{\pi W f_{n}^{0}} G\left(\lambda_{n}^{0}\right) \sqrt{\frac{E}{12 \rho}}$

This approximation is confirmed by FEM simulations. In this approach, the dependence of the Young's modulus on the temperature is neglected. As long as this dependence is small (with an average coefficient of $-63 \mathrm{ppm} / \mathrm{K}$ for silicon [13]) its contribution to the sensitivity can be easily taken into account by adding it to the right hand side of Eq. (6). In order to isolate the effect of axial stress, $E$ was taken constant in the FEM simulations presented in the next section.

\section{Fem simulations}

Finite element simulations of the dependence of the resonance frequency on the temperature were performed. To allow an easy comparison with Eq. (6), the analyses were focused on the effect of the axial stress caused by the temperature and neglected other temperature effects, like Young's modulus changes.

All the analyses were executed in FEMLAB ${ }^{\circledR}$ : in a first step a temperature load was applied, a static nonlinear analysis was done and the consequent stress and strain configuration was stored; then this static solution was loaded for a pre-stressed modal analysis; the operation was repeated for different temperatures and for every kind of resonator in order to find in each case the resonance frequency at different temperatures/axial stresses, and for different width in the case of the $\mathrm{CC} 1$ beam.

In Fig. 6, a comparison between analytical model and simulations for a $\mathrm{CC} 1$ resonator is shown: while the error is acceptable, it is shown to increase for increasing beam thickness, an effect which is not accounted for by the analytical model. In Fig. 7, a comparison between the CC1 resonance frequency stability with respect to the beam width

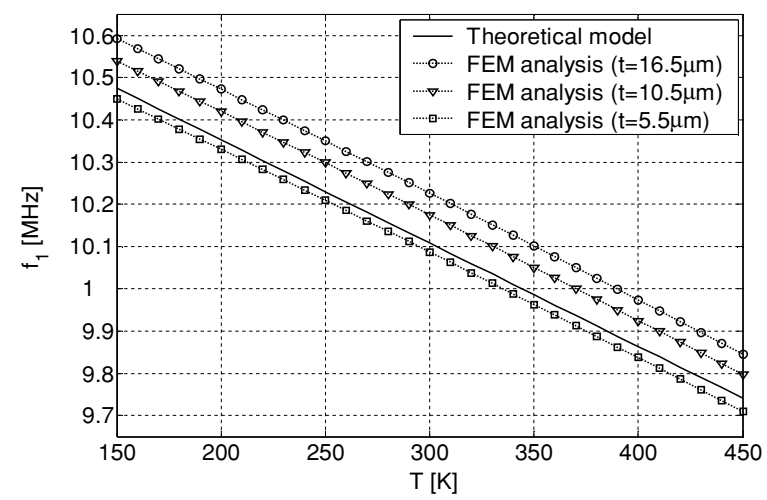

Fig. 6 Simulated and theoretical dependence of the resonance frequency on the temperature for a $\mathrm{CC} 1$ resonator (Substrate temperature $T_{\text {sub }}=300 \mathrm{~K} ; \alpha_{m}=4.5 \mathrm{ppm}$ ) for different beam thicknesses

according to $\mathrm{FEMLAB}^{\circledR}$ simulations and theoretical model (6) respectively, is presented.

The theoretical analysis for $\mathrm{CC}$ resonators leading to Eq. (6) gives interesting suggestions for the design of CC resonators: first, slender beams are more sensitive to axial loads (due to both temperature and/or residual stress) (Fig. 7); moreover, resonators with higher resonance frequencies show a better stability; finally, because $G\left(\lambda_{n}^{0}\right)$ increases with increasing $n$, lower order $\mathrm{CC}$ resonant modes are expected to be more stable than upper ones.

The situation for FF1 and FF3 resonators is complicated by the presence of the supports, which are $\mathrm{CC}$ beams (resonating on their second mode) themselves. A comparison between the simulation results for the three resonator types is shown in Fig. 8. The resonance frequency was simulated for three devices with the same nominal resonance frequency $(10 \mathrm{MHz})$ at $300 \mathrm{~K}$, and the same width $(2.2 \mu \mathrm{m})$. The average thermal expansion cofficient used was $\alpha_{m}=4.5 \mathrm{ppm}$.

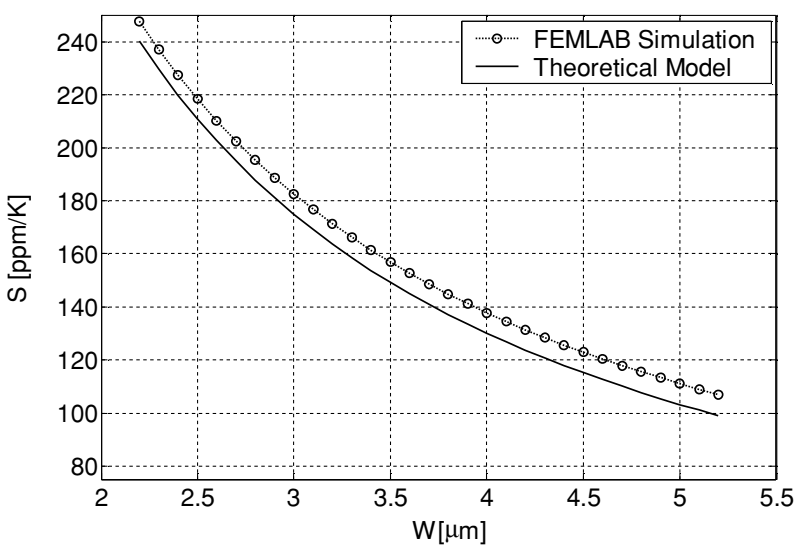

Fig. 7 Simulated and theoretical stability of the resonance frequency of a $\mathrm{CC} 1$ resonator with respect to the temperature difference between resonator and substrate for different beam widths (Substrate temperature $T_{\text {sub }}=300 \mathrm{~K} ; \alpha_{m}=4.5 \mathrm{ppm}$ ); resonance frequency of the unstressed structure $10 \mathrm{MHz}$ (resonator lenght is adjusted to obtain the same frequency at each width) 


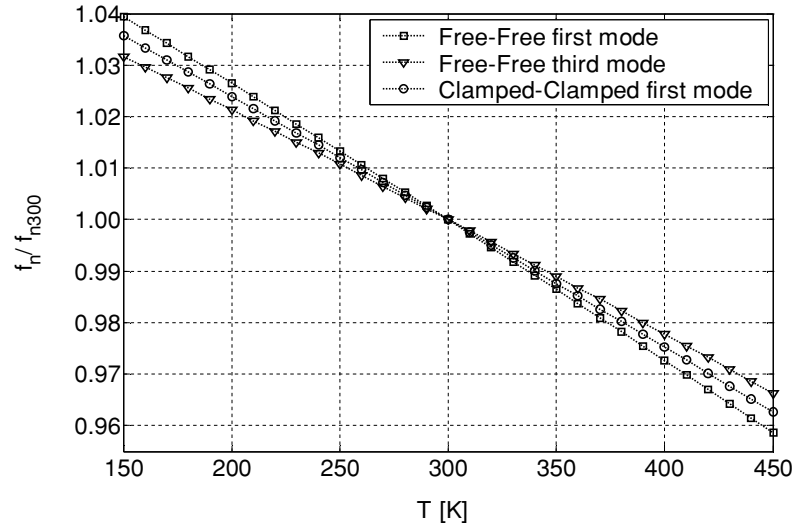

Fig. 8 FEMLAB simulations of the dependence on the temperature of the normalized resonance frequencies for a $\mathrm{CC} 1$, an $\mathrm{FF} 1$ and an FF3 resonator (normalization is done with respect to the resonance frequency at $T=300 \mathrm{~K}) . S=269 \mathrm{ppm} / \mathrm{K}$ for the FF1 resonator, $S$ $=244 \mathrm{ppm} / \mathrm{K}$ for the $\mathrm{CC} 1$ resonator and $S=218 \mathrm{ppm} / \mathrm{K}$ for the FF3 resonator. (Substrate temperature $T_{\text {sub }}=300 \mathrm{~K} ; \alpha_{m}=4.5 \mathrm{ppm}$ )

The worst performance (largest $\Delta f$ on the selected temperature range, or worst stability $S$ ) is that of a free-free resonator, namely the FF1 type. The FF3 resonator has the best performance, while the $\mathrm{CC} 1$ type performs between the two. It is interesting that a higher order FF mode is more stable that a lower order one: this is just the opposite of the behavior predicted by Eq. (6) for CC modes.

An interpretation of the results for FF resonators is not straightforward: in particular, while the main beam in these two cases can be supposed to be axially unloaded (it can easily expand, discharging any axial stress), the frequency shift cannot be ascribed (at least not completely) to the frequency shift of the supports. This has been verified by separate simulations, which show that $\mathrm{CC} 2$ (clamped-clamped second mode) beams of the appropriate length have a much better stability when on their own, i.e. when not connected with the main beam.

\section{Measurements}

To allow characterization of the fabricated devices at different pressure levels, the chip was mounted on a PCB board, containing driving and sensing electronics, and placed in the chamber of a vacuum system, based on a combined membrane pump/turbomolecular pump. The pressure was measured with a standard thermocouple gauge. The investigated pressures were between 2 and $4 \mathrm{~Pa}$.

The 2nd harmonic method [14] was implemented so as to reduce the effect of the parasitic capacitive feed-through between input and output of the devices under test. A sinusoidal input signal $V_{\text {in }}$ at frequency $f / 2$ is applied to the driving electrode, the beam is grounded, and the sensing electrode is biased at a constant voltage $V_{\text {bias }}$. Because of

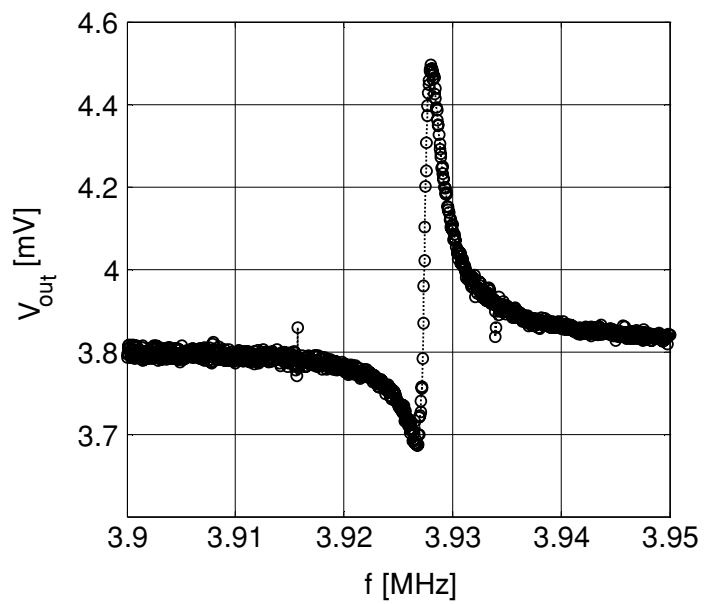

Fig. 9 Measured frequency response of an FF1 device with nominal resonance frequency $5 \mathrm{MHz}$ (Pressure $2.5 \mathrm{~Pa}$, amplitude of the input signal $\left.V_{\text {in }}=1 \mathrm{~V}, V_{\text {bias }}=12 \mathrm{~V}\right)$. Estimated $Q=1600$

the quadratic relationship between the driving voltage and the actuation force, the force (and, consequently, the beam displacement) can be written as a sum of a static component and a sinusoid at frequency $f$. The sinusoidal displacement at frequency $f$ leads to an output current component at the same frequency, while the frequency of the parasitic signal is $f / 2$, so that the two components can be separated by the measurement system.

At each input frequency, the output current is electronically converted to a voltage and amplified to give a signal $V_{\text {out }}$, which is then acquired by a 14-bit, $100 \mathrm{MSa} / \mathrm{s}$ acquisition board. The amplitude of the component at twice the input frequency was then extracted from the FFT spectrum of the output signal.

In Figs. 9 and 10, curves of the frequency response of an FF1 and an FF3 resonator are presented. The large signal floor is caused both by the noise and by the distortion introduced by the electronic amplification chain between the resonator output port and the acquisition board.

The nominal resonance frequency is $5 \mathrm{MHz}$ for the FF1 and $10 \mathrm{MHz}$ for the FF3 resonator, while the measured ones are 3.955 MHz (Fig. 9) and $8.537 \mathrm{MHz}$ (Fig. 10), respectively. This mismatch can be attributed mostly to non-ideal behavior of the anchors, which were considered perfectly rigid in the simplified design.

Because of the aforementioned signal floor, the quality factor $Q$ of the resonators could only be estimated. The curves were fitted with a function expressed as the sum of a 2nd order resonant system response and of a component due to the capacitive current (to take into account the 2nd order distortion of the parasitic feed-through caused by the amplification chain). The fittings are quite good for the presented measurements and give a $Q$ of about 1600 for the FF1 and about 5000 for the FF3 device. 


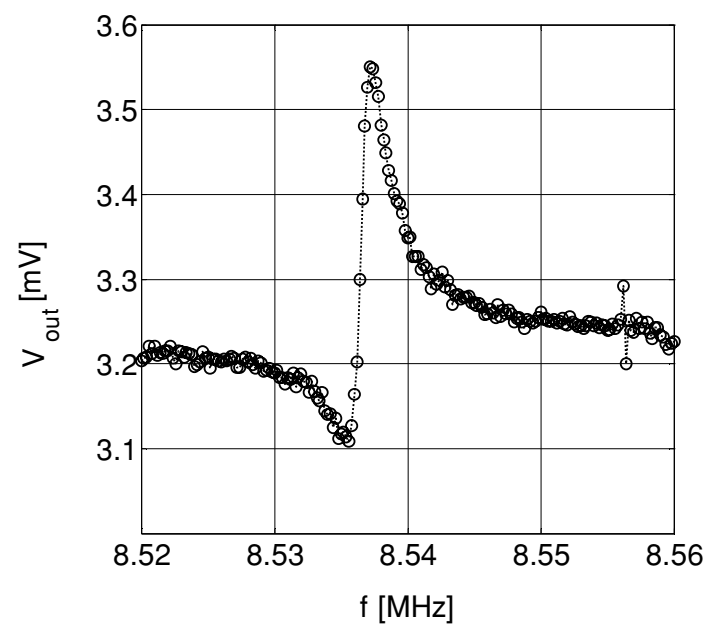

Fig. 10 Measured frequency response of an FF3 device with nominal resonance frequency $10 \mathrm{MHz}$ (Pressure $4 \mathrm{~Pa}$, amplitude of the input signal $\left.V_{\text {in }}=1 \mathrm{~V}, V_{\text {bias }}=15 \mathrm{~V}\right)$. Estimated $Q=5000$

\section{Conclusions}

Three different kinds of resonators were designed and fabricated, and their stability with respect to temperature and residual stress was investigated both theoretically and by FEM simulations. The results can be used as interesting guidelines for the design of frequency-stable MEMS resonators. FEM simulations showed that third mode free-free resonators have the best frequency stability behavior. The basic operation of this novel free-free third mode device was also demonstrated. To verify the effect of temperature on the frequency stability of the proposed resonators, further measurements are planned.

Acknowledgments STMicroelectronics fabricated the resonators at their Cornaredo (Milan) plant, in the framework of the joint University of Pisa-STMicroelectronics Microsystems R\&D center.

\section{References}

1. C.T.-C. Nguyen, "Transceiver front-end architectures using vibrating micromechanical signal processors." In Proceeding of Topical Meeting on Silicon Monolithic Integrated Circuits in RF Systems, Ann Arbor, MI, USA, 2001, pp. 23-32.

2. T. Mattila, J. Kiihamäki, T. Lamminmäki, O. Jaakkola, P. Rantakari, A. Oja, H. Seppä, H. Kattelus, and I. Tittonen, "A $12 \mathrm{MHz}$ micromechanical bulk acoustic mode oscillator." Sensors and Actuators A: Physical, vol. 101, pp. 1-9, 2002.

3. S. Lee, M.U. Demirci, and Clark T.-C. Nguyen, "A $10 \mathrm{MHz}$ micromechanical resonator Pierce reference oscillator for communications." In Proceeding of 11th Int. Conf. on Solid-State Sensors \& Actuators (Transducers'01), Munich, Germany, 2001, pp. 10941097.

4. M. Koskenvuori, T. Mattila, A. Häärä, J. Kiihamäki, I. Tittonen, A. Oja, and H. Seppä, "Long-term stability of single-crystal silicon microresonators." Sensors and Actuators A: Physical, vol. 115, pp. 23-27, 2004.
5. W.-T. Hsu, J.R. Clark, and C.T.-C. Nguyen, "Mechanically temperature-compensated flexural-mode micromechanical resonators." In Proceeding of Electron Devices Meeting, San Francisco, CA, USA, 2000, pp. 399-402.

6. W.-T. Hsu, J.R. Clark, and C.T.-C. Nguyen, " $Q$-optimized lateral free-free beam micromechanical resonators.", In Proceeding of 11th Int. Conf. on Solid-State Sensors \& Actuators (Transducers'01), Munich, Germany, 2001, pp. 1110-1113.

7. D. Galayko, A. Kaiser, B. Legrand, L. Buchaillot, C. Combi, and D. Collard, "Clamped-clamped beam micro-mechanical resonators in thick-film epitaxial polysilicon technology." In Proceeding of ESSDERC2002, Firenze, Italy, 2002, pp. 447-450.

8. M.U. Demirci and C.T.-C. Nguyen, "Higher-mode free-free beam micromechanical resonators." In Proceeding of Frequency Control Symposium and PDA Exhibition Jointly with the 17th European Frequency and Time Forum Piscataway, NJ, USA, 2003, pp. 810818.

9. http://www.comsol.com/products/femlab/.

10. E. Quevy, D. Galayko, B. Legrand, C. Renaux, C. Combi, D. Flandre, L. Buchaillot, D. Collard, B. Vigna, and A. Kaiser, " IF MEMS filters for mobile communication." In Proceeding of 8th IEEE International Conference on Emerging Technologies and Factory Automation, Antibes, France, 2001 vol. 2, pp. 733-736.

11. H.A.C. Tilmans, "Equivalent circuit representation of electromechanical transducers: II. Distributed-parameter systems." Journal of Micromechanics and Microengineering, 1997, vol. 7, pp. 285309.

12. H.A.C. Tilmans, M. Elwenspoek, and J.H.J. Fluitman, "Micro resonant force gauges." Sensors and Actuators A: Physical, vol. 30, pp. 35-53, 1992.

13. M.J. Madou, Fundamentals of Microfabrication, 2nd edn, CRC Press, Boca Raton, FL, USA, 2002.

14. P. Bruschi, A. Nannini, D. Paci, and F. Pieri, "A method for crosssensitivity and pull-in voltage measurements of MEMS two-axis accelerometers." Sensors and Actuators A: Physical, vol. 123-124, pp. 185-193, 2005.

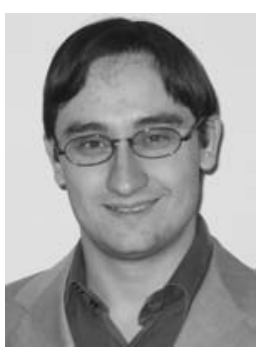

Dario Paci studied electronic engineering at University of Pisa and at "Scuola Superiore Sant'Anna". He received his Master Degree in 2003, with a dissertation on MEMS resonators for RF applications. In 2003 he worked at PEL-ETHZ for three months, modelling chemical sensors. In 2005 he was visiting scholar at the Katholieke Universiteit Leuven (Belgium), working at IMEC on MEMS resonators anchor losses modeling. His research interests include MEMS modelling and design and development of circuits for MEMS conditioning. Now he is pursuing the Ph.D. in Information Engineering at University of Pisa, and he is working as a research assistant for the IEIIT of the Italian National Council for the Research (CNR).

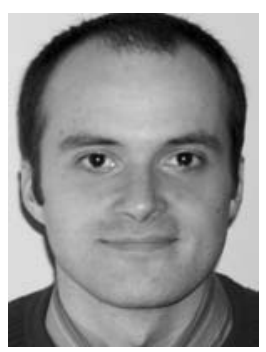

Massimo Mastrangeli got the MS degree in Electronic Engineering at the University of Pisa (Italy) on July, 2005; his thesis concerned project and measurements of MEMS flexural resonators. During summer 2005 he was visiting scholar at the Katholieke Universiteit Leuven (Belgium), working at IMEC on the mechanical characterization of PolySiGe layers for MEMS applications. He is currently a $\mathrm{PhD}$ student at KULeuven, developing a techniques for self-assembly of IC/MEMS for highly integrated microsystems. 


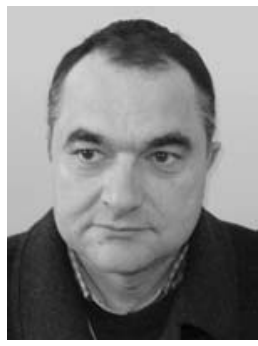

Andrea Nannini received his laurea degree in Electronic Engineering from the University of Pisa, Italy, in 1982; He received his $\mathrm{Ph} . \mathrm{D}$ degree in 1987 at the end of the first Italian Ph.D course held by the University of Padova, Italy. From 1988 to 1992 he was a Researcher at the "Scuola Superiore di Studi Universitari e Perfezionamento S. Anna"-Pisa- Italy. Since 1992 he joined the Department of Information Engineering of the University of Pisa as an Associate Professor. Since November 2000 he is a full professor of "Sensor and Microsystem Design". $\mathrm{He}$ is currently chairman of the postgraduate school of Electronic Engineering and vice-chairman of the $\mathrm{PhD}$ school of Information
Engineering of the University of Pisa. His main research interests concern solid state sensors, microelectronic devices and technologies, MEMS.

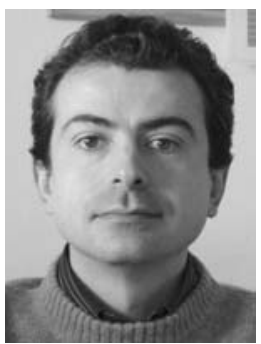

Francesco Pieri received the laurea and the Ph.D. degree in Electrical Engineering, both from the University of Pisa, Italy, in 1996 and 2000 respectively. He joined the Department of Information Engineering of the same University as an assistant professor in 2001. His current research interests include applications of porous silicon to sensors and microtechnologies, and development of microelectromechanical systems. 